\title{
Density Distribution at the Detonation Front of Cylindrical Charges of Small Diameter
}

\author{
K. A. Ten, ${ }^{1}$ O. V. Evdokov, ${ }^{2}$ I. L. Zhogin, ${ }^{2}$ \\ V. V. Zhulanov, ${ }^{3}$ P. I. Zubkov, ${ }^{1}$ G. N. Kulipanov, ${ }^{3}$ \\ L. A. Luk'yanchikov, ${ }^{1}$ L. A. Merzhievskii, ${ }^{1}$

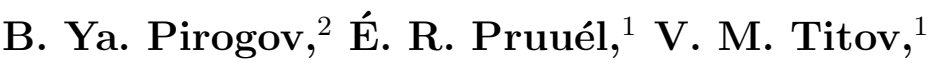 \\ B. P. Tolochko, ${ }^{2}$ and M. A. Sheromov ${ }^{3}$
}

UDC 534.222 .2

Translated from Fizika Goreniya i Vzryva, Vol. 43, No. 2, pp. 91-99, March-April, 2007.

Original article submitted May 17, 2006.

\begin{abstract}
A synchrotron radiation based technique is use to study the density distribution at the detonation front and its neighborhood for condensed explosives. Particular data are obtained on the structure of the detonation front in TNT, RDX, and an alloy of TNT with RDX; a comparison of the data with those obtained using different techniques confirms the correctness of the technique. It is concluded that adequate information on the structure of the chemical-reaction zone can be obtained for charges of small diameter. At the same time, it is shown that the Chapman-Jouguet parameters for such charges are far from their predicted values for an infinite medium. The results of the work, including those on the curvature of the detonation front in charges of small diameter, supplement the existing knowledge of the detonation transformation in condensed explosives.
\end{abstract}

Key words: explosives, synchrotron radiation, detonation, front structure.

\section{INTRODUCTION}

The modern concepts of the steady-state detonation of high explosives (HEs) are based on the hydrodynamic Michelson-Chapman-Jouguet (MCJ) model and its extension - the Zel'dovich-Neumann-Döring (ZND) model. According to the latter, steady-state detonation is a self-sustained wave process. The detonation wave consists of a shock wave, which initiates chemical reaction, and an exothermic-reaction zone, which sustains the leading shock wave and follows it immediately or with a delay. The reaction zone is terminated by the Chapman-Jouguet plane, in which chemical equilibrium

\footnotetext{
${ }^{1}$ Lavrent'ev Institute of Hydrodynamics,

Siberian Division, Russian Academy of Sciences, Novosibirsk 630090; merzh@hydro.nsc.ru.

${ }^{2}$ Institute of Solid State Chemistry and

Mechanochemistry, Siberian Division, Russian Academy of Sciences, Novosibirsk 630090.

${ }^{3}$ Budker Institute of Nuclear Physics, Siberian Division, Russian Academy of Sciences, Novosibirsk 630090.
}

is reached and the flow relative to the front is sonic. The values of the parameters (Chapman-Jouguet parameters) characterizing the initial state of detonation products refer to this plane.

A wide range of experimental methods based on various physical principles has been employed to determine the Chapman-Jouguet parameters, steady-state detonation regimes, and the structure of the chemicalreaction zone. The first published experimental data on these parameters were apparently obtained from measurements of the velocity of an aluminum target in contact with a HE charge [1]. Similar methods were developed independently by Soviet researchers [2]. A conditional division of all method of investigation of detonation processes into external and internal methods was proposed in [3]. A common disadvantage of the external methods, which are based on measuring the velocity of the free surface at the moment the detonation wave arrives at this surface, is that the detonation parameters have to be calculated using the model that should be 
constructed or refined by the experiments performed. The internal methods, which include flash radiography of foils moving inside a charge, pressure measurements by Manganin gauges, and measurements of particle velocity with electromagnetic gauges, allow one to directly record detonation parameters but introduce perturbations to the process studied.

The results of numerous observations basically agree with the ZND model but there is some uncertainty in estimating the reliability and interpreting available experimental data. The reasons of the differences in the Chapman-Jouguet parameters for a number of HEs measured using identical techniques are a subject of discussion. A critical analysis of the measurement methods and the reliability of the results obtained is given, for example, in $[3,4]$. One of the most debatable problems is the correctness of experimental data on the structure of the chemical-reaction zone and its relationship to the detonation transformation kinetics. Of fundamental significance here is the question of the absence of a chemical spike in some cases, which is a necessary element of the detonation wave front in the ZND model. This was first reported in [5]. Later, a cycle of papers with similar results appeared [6-8], but the reliability of these data has been debated (see., for example, [4]). A criterion of reliability in this case can be the collection of data on the detonation process using different techniques, including internal unperturbed methods and their comparison. Agreement between data obtained with different measurement techniques is a powerful argument for the their correctness.

The density distribution in a detonating material can be measured directly using synchrotron radiation [9]. A similar method of studying detonation using a proton beam, together with some results, is described in $[10,11]$. The present paper gives results of measurements of the density distribution during detonation of cylindrical charges using the method of [9]. These data provide, in particular, information on the structure of the reaction zone at the detonation front.

\section{EXPERIMENTAL TECHNIQUE}

Synchrotron radiation (SR) is used in an unperturbed internal method to measure the parameters of a detonating $\mathrm{HE}$ charge in a region adjacent to the detonation front, including (under certain conditions) the front itself. A detailed description of the capabilities of $\mathrm{SR}$ in studies of detonation and shock-wave processes is given in [9]; therefore, here we shall consider only the particular experimental technique.

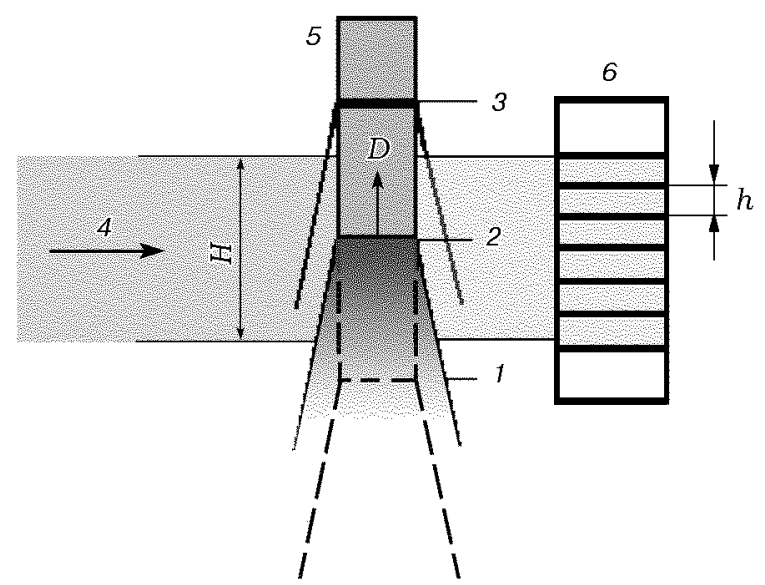

Fig. 1. Experimental setup: positions of the detonation front at various times (1-3), SR beam (4), HE charge (5), and detector (6).

The density distribution for detonation of cylindrical charges of pressed TNT, RDX, and 50/50 TNT/RDX alloy of relatively small diameter was studied. Before pressing, all HEs were recrystallized using the well-known technique with acetone to improve the quality of the charges. The diameter of the pressed HE charges was $12.5-15 \mathrm{~mm}$, the diameter of the cast charges was $7-12.5 \mathrm{~mm}$, and the length of all charges was 8-10 times the diameter, which ensured that the detonation process was steady-state. Charges of diameter exceeding $12.5 \mathrm{~mm}$ were initiated by plane-wave generators (PWG).

The experimental setup is shown in Fig. 1. The HE charge was placed horizontally and perpendicular to the SR beam $0.4 \mathrm{~mm}$ high and $H=18 \mathrm{~mm}$ wide. The plane of the SR beam passed along the axis of the examined HE charge, and the flash duration was $10^{-9} \mathrm{sec}$ at a hardness of $10-30 \mathrm{keV}$. The detonation front (with detonation velocity $D$ ) sequentially passed positions $1-3$, remaining in the zone of the SR beam for 3-4 $\mu \mathrm{sec}$. This allowed us to take $3-5$ photographs of the distribution of the transmitted radiation along the axis of the charge with an exposure of 1 nsec. The time between the photographs was determined by the period of rotation of the electron bunch in the accelerator and was $250 \mathrm{nsec}$. The radiation was recorded by a DIMEX detector 6 [12], which was also placed parallel to the axis of the charge at a distance of $1070 \mathrm{~mm}$ from the HE charge. One recording channel was $1 \mathrm{~mm}$ high and $0.1 \mathrm{~mm}$ along the charge axis, and the total number of channels was 256 ; thus, a segment $25.6 \mathrm{~mm}$ long along the charge was studied. The detector can record 32 frames collected in this fast mode and has 

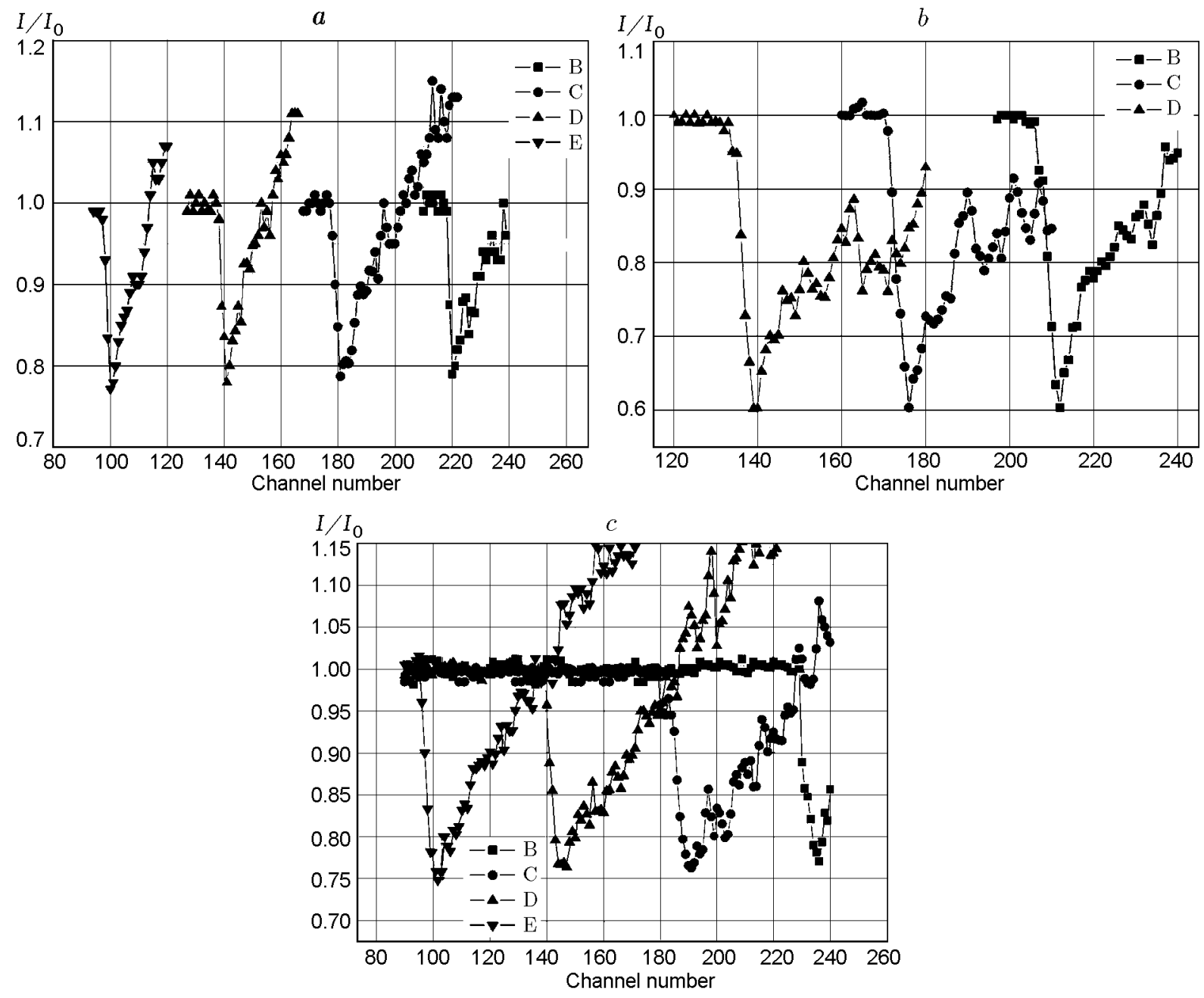

Fig. 2. Radiation intensity distribution after absorption in 50/50 TNT/RDX charges (a), TNT (b), and RDX (c) at times $t=0$ (B), 500 (C), 1000 (D), and $1500 \mathrm{nsec}(\mathrm{E})$.

a recording efficiency $\approx 50 \%$ for $\gamma$-quanta with energies $\approx 30 \mathrm{keV}[12]$. The detector was started by closing the contact gauge placed at $15 \mathrm{~mm}$ behind the zone of incidence of the SR beam.

\section{RESULTS OF EXPERIMENTS AND THEIR INTERPRETATION}

As a result of the experiments, we obtained the intensity distribution of the radiation $I$ transmitted through the examined object along the charge axis at various times. Examples of such distributions in the form of the ratio of $I$ to the intensity of the initial beam $I_{0}$ versus the detector channel number are given in Fig. 2. The process propagates toward decreasing channel number, and the time between the given profiles is $0.5 \mu \mathrm{sec}$.
The design features of the detector used in the experiments are such that when a $\gamma$-ray quantum falls in one of the recording channels, a charge occurs not only in this channel but also in the neighboring channels [12]. This results in spatial spreading of the signal boundaries even in the case where the tested object absorbs the radiation completely. The nature of the spreading is shown in Fig. 3, which gives the distribution of the radiation recorded by the detector (in arbitrary units) on the boundary of a target which completely absorbs the radiation and is on the right of the 243th channel. A marked signal is observed in at least three overlapped channels. The detector accuracy in the region of high density gradients were refined using a specially designed algorithm. The algorithm uses the experimentally determined apparatus broadening function [13]. Figure 4 compares readings of the detector and the result of cor- 


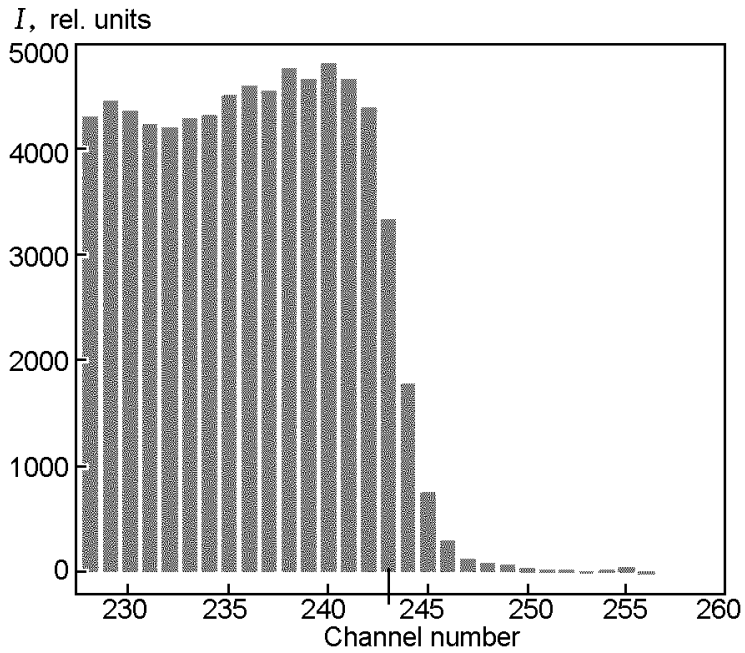

Fig. 3. Radiation intensity distribution at the edge of an opaque object recorded by the detector.

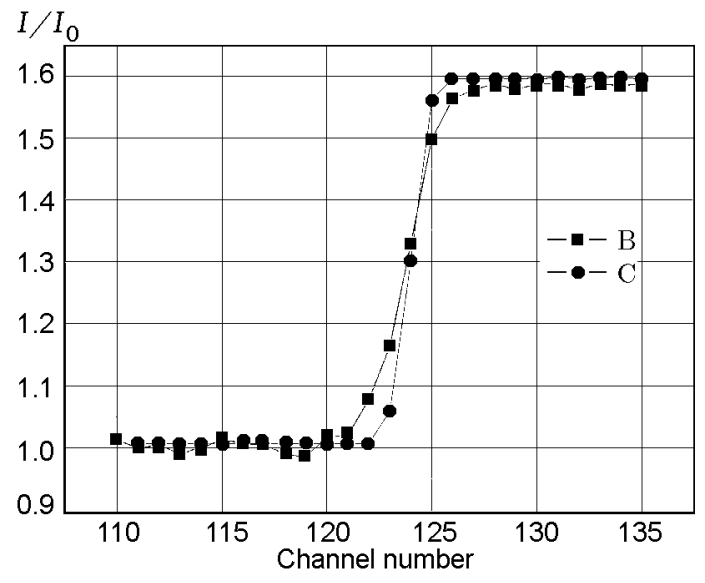

Fig. 4. Comparison of the initial (points B) and corrected (points $\mathrm{C}$ ) distributions of the radiation intensity.

rection of these readings obtained in a test experiment with a polymethyl methacrylate sample. The further test of the capabilities of the correction algorithm by processing a specified signal distorted by superposition of random noise showed that this algorithm allows the original signal to be restored with an accuracy of up to $1 \%[13]$.

From the corrected data on the distribution of the transmitted-radiation intensity (see Fig. 2), it is required to determine the dependence of the density in the neighborhood of the detonation front on the longitudinal coordinate. Generally, the attenuation of the intensity is determined by the multifactor interaction of the radiation with the material: variation in the absorption spectrum due to variation in the material density

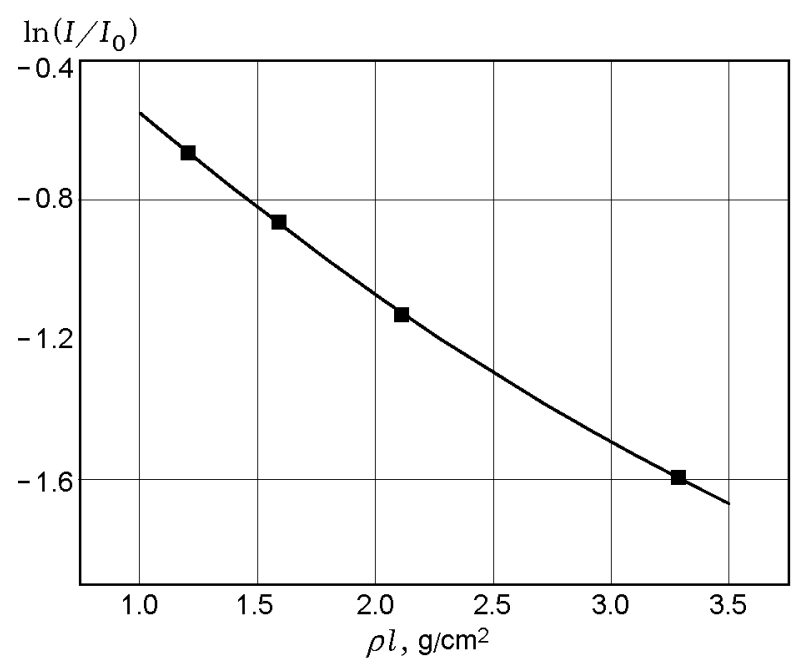

Fig. 5. Calibration dependence of radiation absorption on the amount of the tested material (RDX).

along the beam, the dependence of the absorption coefficient on wavelength, elastic and inelastic scattering, ionization losses, the curving of the beam track on the charge-air boundary and inside the examined sample at density gradients, etc. In addition, the result is affected by the nonlinearity of the detector and its complex spectral response. As a result, even in the case where not all factors are taken into account, the energy of the beam transmitted through an object $L$ and recorded by the detector is given by the relation

$$
J_{L}=\int_{E} J_{0 L}(E) D(E) \exp \left(-\int_{L} \mu(r, E) d r\right) d E,
$$

where $E$ is the X-ray energy, $J_{0 L}(E)$ is the $\mathrm{X}$-ray source energy density along the beam $L, D(E)$ is the coefficient of absorption of radiation with energy $E$ by the detector, and $\mu(r, E)$ is the linear coefficient of absorption of radiation with energy $E$ at the point $r$. To simplify the procedure of finding the amount of the tested material and the density distribution, we assume that the degree of radiation attenuation depends only on the mass of the tested material, i.e., the detector readings $F(x)$ are given by the relation

$$
F(x)=f\left(\int_{L} \rho(x) d l\right),
$$

where the unknown function $f$ is determined experimentally. The dependence of the recorded intensity on the amount of the tested material was constructed from experimental data for each detector channel. Homogeneous plates of various thickness (3-5 measurements) were examined. The results of determination of the function $f$ for RDX are presented in Fig. 5 ( $l$ is the 


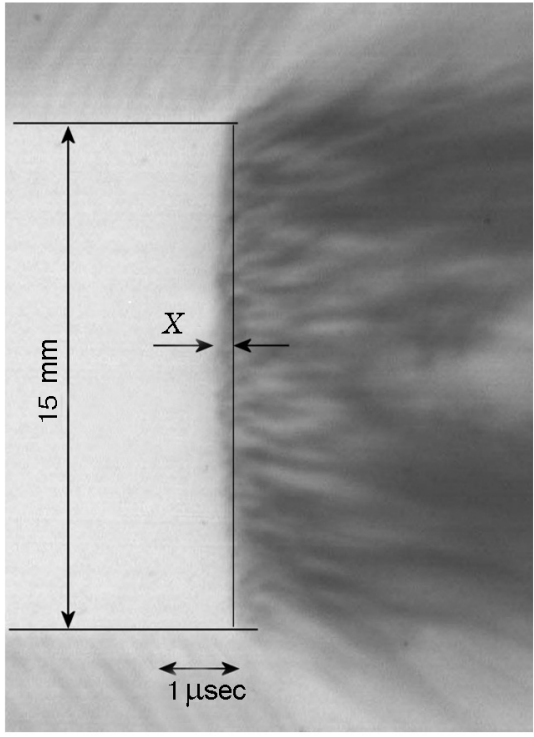

Fig. 6. Streak record of the luminescence at the time the detonation front arrives at the end of a 50/50 TNT/RDX charge.

depth of the radiographed path). The experimental data on the plane $(\ln I, \rho l)$ are well approximated by the parabola

$$
\ln \frac{I}{I_{0}}=-0.678 \rho l+0.051(\rho l)^{2} .
$$

As a first approximation, the dependence can be considered linear with good accuracy. The calibration curve thus constructed was used to determine the quantity of the material along the beam from the measured intensity of the transmitted radiation and then to obtain the density distribution.

The next feature that should be taken into account in interpreting the results is the curvature of the detonation front in practice. The front curvature in the charges studied was determined in specially conducted experiments using high-speed photography of the arrival of the detonation front at the end. A typical streak record is given in Fig. 6 (pressed TNT charge). As a first approximation, the detonation front can be considered part of a sphere; then, the front curvature is conveniently characterized by its radius calculated from experimental data. The values of the curvature radius $R$ and the detonation velocity $D$ are given in Table 1 . In the subsequent calculation of density at a particular point and construction of the spatial density distribution function, the curvature radius was used to calculate the beam length on which radiation absorption occurs, i.e., the value of $l$ in formula (1).
TABLE 1

\begin{tabular}{c|c|c|c|c}
\hline $\mathrm{HE}$ & $d, \mathrm{~mm}$ & $\begin{array}{c}\text { Initiation } \\
\text { method }\end{array}$ & $D, \mathrm{~km} / \mathrm{sec}$ & $R / d$ \\
\hline $\mathrm{TNT}$ & 15 & $\mathrm{PWG}$ & 6.95 & 2.1 \\
\hline $\mathrm{RDX}$ & 15 & - - & 8.3 & 2.35 \\
\hline $\begin{array}{c}50 / 50 \\
\mathrm{TNT} / \mathrm{RDX}\end{array}$ & 7 & Detonator & 7.55 & 1.9 \\
\hline $\begin{array}{c}50 / 50 \\
\mathrm{TNT} / \mathrm{RDX}\end{array}$ & 12.5 & $-1 /-$ & 7.67 & 2.04 \\
\hline
\end{tabular}

\section{DISCUSSION OF RESULTS}

Detonation processes are usually studied with charges of fairly large diameter, far exceeding the limiting one. In the present work, the charge diameters exceeded the critical diameters of the explosives studied but they might be comparable to the limiting values. This can lead to a difference between the characteristics of the processes studied and their standard values for the given HEs. This refers primarily to the velocity and curvature of the detonation front, and, hence, the remaining Chapman-Jouguet parameters. Let us consider the results of the tests performed from this point of view.

The measured detonation velocity for $50 / 50$ TNT/RDX charges is $D=7.55 ; 7.63$, and $7.65 \mathrm{~km} / \mathrm{sec}$ for charges of diameter $d=7,10$, and $12.5 \mathrm{~mm}$, respectively. In a charge of diameter $7 \mathrm{~mm}$, the detonation velocity increases from 7.4 to $7.55 \mathrm{~km} / \mathrm{sec}$. As the charge diameter increases, the curvature of the front (see Table 1) decreases. The gradual increase in the velocity up to the value standard for the given density [14] implies that at least the diameter $d=12.5 \mathrm{~mm}$ is not smaller than the limiting one. Although data on the front curvature in 50/50 TNT/RDX charges of diameter far exceeding the limiting value are not available, the value obtained in this work agrees with the estimates that can be derived from the relations given, for example, in [15]. The profiles given in Fig. 2 indicate that even in the case of the smallest diameter, the process is nearly steady-state. The aforesaid suggests that the obtained data refer to steady-state detonation propagation and the observed features are not related to geometrical factors.

The critical diameter of TNT is $5-10 \mathrm{~mm}$, depending on the initial particle size [14]. The measured detonation velocity almost coincides with that given in [14]. This and a comparison of the front curvature with literature data, together with the data of Fig. $2 \mathrm{~b}$ indicating a steady-state process, suggest that the characteristics obtained are characteristics of the process for TNT, too. 

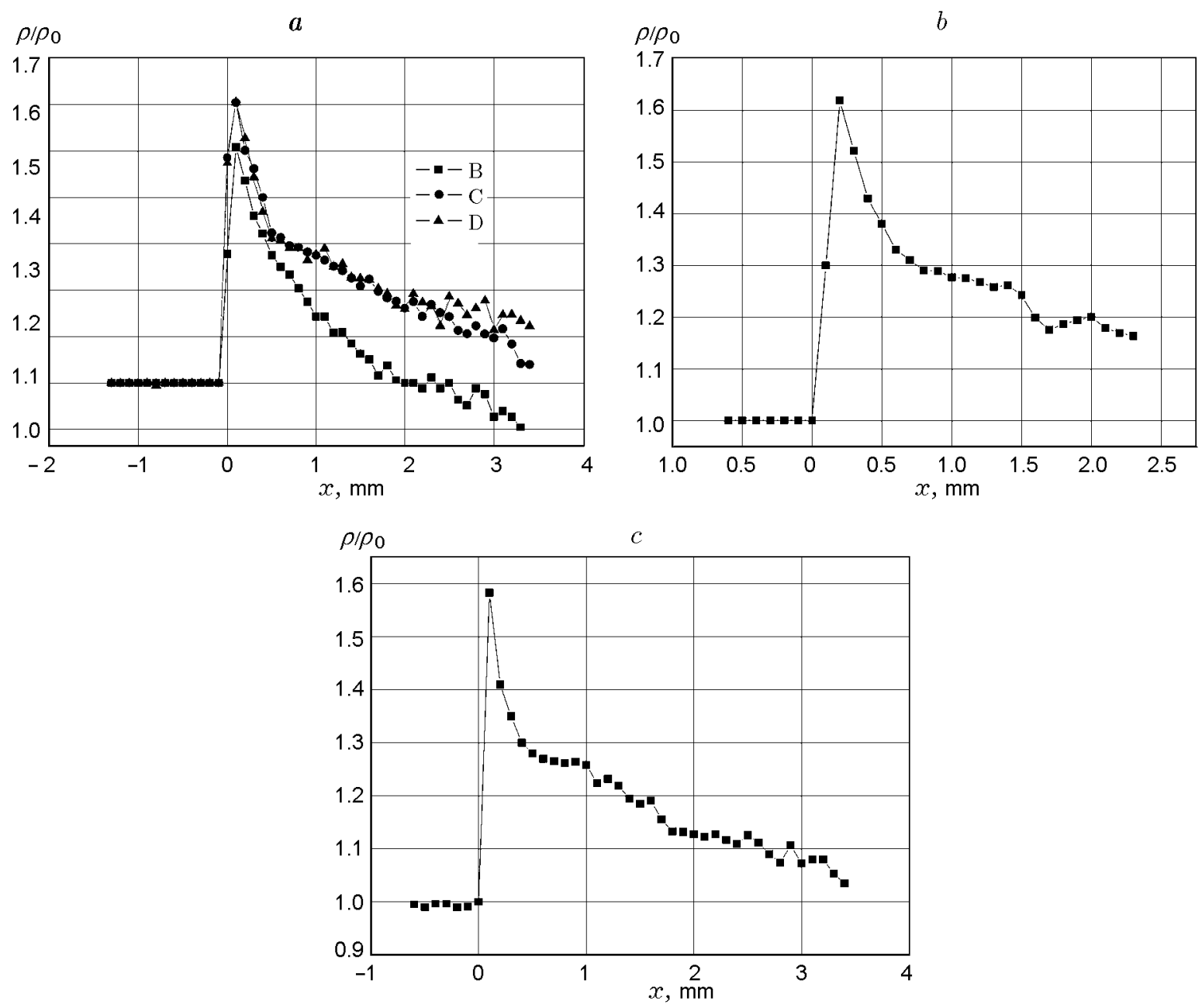

Fig. 7. Density distribution for detonation of 50/50 TNT/RDX (a), TNT (b), and RDX (c) charges: (a) $d=7$ (B), $10(\mathrm{C})$, and $12.5 \mathrm{~mm}(\mathrm{D}) ;(\mathrm{b}, \mathrm{c}) d=15 \mathrm{~mm}$.

TABLE 2

\begin{tabular}{l|c|c|c|c|c|c|c|c}
\hline $\mathrm{HE}$ & $\rho_{\mathrm{N}}, \mathrm{g} / \mathrm{cm}^{3}$ & $\rho_{\mathrm{CJ}}, \mathrm{g} / \mathrm{cm}^{3}$ & $\tau, \mu \mathrm{sec}$ & $\Delta, \mathrm{mm}$ & $\Delta_{1}, \mathrm{~mm}$ & $\gamma$ & $\rho_{\mathrm{N}} / \rho_{\mathrm{CJ}}$ & $K$ \\
\hline $50 / 50 \mathrm{TNT} / \mathrm{RDX}$ & 2.64 & 2.16 & $0.065 \pm 0.013$ & $0.5 \pm 0.1$ & $0.1-0.8$ & 3 & 1.22 & 1.81 \\
\hline $\mathrm{TNT}$ & 2.61 & 2.09 & $0.1 \pm 0.014$ & $0.7 \pm 0.1$ & $0.63-0.97$ & 3.3 & 1.25 & 2.09 \\
\hline $\mathrm{RDX}$ & 2.6 & 2.26 & $0.048 \pm 0.012$ & $0.4 \pm 0.1$ & $0.36-0.6$ & 3.1 & 1.15 & 1.54 \\
\hline
\end{tabular}

In the case of RDX charges, the situation is not as unambiguous. The data of Fig. $2 \mathrm{c}$ show that as the detonation propagates, both the shape of the absorption (density) profile and the maximum density value at the detonation front vary. In this case, the measured detonation velocity is somewhat smaller than the standard value for the given density [14]. Since the critical diameter of RDX is small, in this case one probably ob- serves the late stage of the transition process related to the establishment of steady-state detonation after initiation.

The density profiles obtained as described above are shown in Fig. 7. The data given there are highly informative and can be used to determine the parameters of the Neumann spike and the parameter values in the Chapman-Jouguet plane. It should be born in 
mind that the steady-state value of the Neumann spike is obtained for charge diameters approximately equal to the critical diameter [16], whereas the parameter values in the Chapman-Jouguet plane continue to increase with increasing charge diameter and in the process of detonation-wave propagation in the HE [16, 17].

The density at the Neumann spike is determined from the data of Fig. 7 in an obvious fashion. The question of its width (the size of the chemical-reaction zone) is more complicated. Originally, the position of the Chapman-Jouguet plane and, hence, the distance between the shock front and this plane was determined from the break of the curve of the measured parameter (particle velocity, velocity of the free surface in a thin plate bordering the charge studied, or the velocity of the shock wave generated in an inert target with known shock adiabat). It turns out, however, that in some cases, the corresponding curves have two breaks (for this see, for example, [14]), resulting in errors in determining the parameters, or a distinct break is absent [5]. A more reliable method is the one in which the point corresponding to the Chapman-Jouguet state is determined as the point at which the segments of the curves corresponding to self-similar rarefaction waves are intersected with superimposition of the curves obtained for charges of different diameters $[3,5,14]$. This approach can be implemented using the data for $50 / 50$ TNT/RDX alloy (see Fig. 7a), for which the ChapmanJouguet state is determined with high reliability. The parameters of the detonation front for TNT and RDX were calculated from the break point on the density profiles, and their reliability was checked by comparing with literature data obtained using different techniques.

The characteristics of the detonation-front structure calculated from the data of Fig. 7 are given in Table 2. Here $\rho_{\mathrm{N}}$ is the density at the maximum of the Neumann spike, $\tau$ and $\Delta$ are its duration and width, respectively, $\rho_{\mathrm{CJ}}$ is the density in the Chapman-Jouguet plane, and $\gamma$ is the polytropic exponent of the products in the Chapman-Jouguet plane. The sixth column (for $\left.\Delta_{1}\right)$ gives generalized literature experimental data on the size of the chemical-transformation zone obtained by various methods, which are fairly completely listed in $[6,14,18]$. The parameter $K$, introduced in [16], characterizes the pressure ratio at the Neumann spike and the Chapman-Jouguet plane. In our case, it was calculated from the ratio $\rho_{\mathrm{N}} / \rho_{\mathrm{CJ}}$ and the polytropic exponent $\gamma$.

A comparison of $\Delta$ and $\Delta_{1}$ parameters shows that the parameters of the reaction zone determines using SR agree with the corresponding data obtained by different methods. According to the data of [16], in most highdensity solid HE charges of large diameter and length,
$K=1.3 \pm 0.1$. The obtained ratio of the parameters at the Neumann spike and in the Chapman-Jouguet plane reflects the fact that the investigation was performed on charges of relatively small diameter since, according to the data of $[16,17]$ and other researchers, the charge size has a significant effect on the effective pressure in the Chapman-Jouguet plane.

\section{CONCLUSIONS}

A synchrotron radiation technique for studying the density distribution at the detonation front and its neighborhood for condensed HEs was described. The technique includes algorithms to correct detector readings, determine the quantity of the material subjected to the synchrotron radiation beam, and find the density distribution with the curvature of the detonation front taken into account. Particular data were obtained on the structure of the detonation front in TNT, RDX, and 50/50 TNT/RDX alloy; their comparison with similar data obtained using other techniques confirms the validity of the proposed technique. The analysis performed leads to the conclusion that adequate information on the structure of the chemical-reaction zone can be obtained in studies using charges of small diameter. At the same time, it is shown that the Chapman-Jouguet parameters for such charges are far from their predicted values for an unbounded medium. The results of the work, including the data on the curvature of the detonation front in charges of small diameter, supplement the current knowledge on the detonation transformation in condensed HEs.

The technique as a whole and the results obtained can be useful in testing, refining, and constructing new equations of the kinetics of detonation transformations and the equations of state of detonation products. It should be noted that the method is also suitable for studies of the behavior of shock-loaded low-density inert media, which are described, for example, in [19].

This work was supported by Integration project of the Siberian Division of the Russian Academy of Sciences, (No. 120), the Foundation "Leading Scientific Schools" (Grant No. NSh 2073.2003.1), and the Russian Foundation for Basic Researches (Grant Nos. 0503-32752 and 06-02-17335).

\section{REFERENCES}

1. R. E. Duff and E. J. Houston, "Measurement of the Chapman-Jouguet pressure and reaction zone length in a detonating high explosive," J. Chem. Phys., 23, No. 7, 1268-1273 (1955). 
2. L. V. Al'tshuler, K. K. Krupnikov, B. N. Ledenev, et al., "Dynamic compressibility and the equation of state of iron at high pressures," Zh. Éksp. Tekh. Fiz., 34, No. 4, 847-885 (1958).

3. L. V. Alt'shuler, G. S. Doronin, and V. S. Zhuchenko, "Detonation regimes and Jouguet parameters of condensed explosives," Combust., Expl., Shock Waves, 25, No. 2, 209-224 (1989).

4. K. K. Shvedov, "Some detonation problems of condensed explosives," Khim. Fiz., 23, No. 1, 27-49 (2004).

5. V. K. Ashaev, G. S. Doronin, and A. D. Levin, "Detonation front structure in condensed explosives," Combust., Expl., Shock Waves, 24, No. 1, 88-92 (1988).

6. B. K. Loboiko and S. N. Lyubyatinskii, "Reaction zones of detonating solid explosives," Combust., Expl., Shock Waves, 36, No. 6, 716-733 (2000).

7. A. V. Utkin, S. A. Kolesnikov, and V. E. Fortov, "Structure of a steady-state detonation wave in pressed RDX," Dokl. Akad. Nauk, 381, No. 6, 760-762 (2001).

8. A. V. Utkin, S. A. Kolesnikov, and S. V. Pershin, "Effect of the initial density on the structure of detonation waves in heterogeneous explosives," Combust., Expl., Shock Waves, 38, No. 5, 590-597 (2002).

9. A. N. Aleshaev, P. I. Zubkov, G. N. Kulipanov, et al., "Application of synchrotron radiation for studying detonation and shock-wave processes," Combust., Expl., Shock Waves, 37, No. 5, 585-593 (2001).

10. N. S. P. King, E. Ables, K. Adams, et al., "An $800-\mathrm{MeV}$ proton radiography facility for dynamic experiments," Nucl. Instrum. Meth. Phys. Res. A, 424, 84-91 (1999).
11. E. N. Ferm, S. Dennison, R. Lopez, et al., "Proton radiography experiments on shocked high explosive products," in: Shock Compression of Condensed Material (2003), pp. 839-842.

12. V. Aulchenko, O. Evdokov, S. Ponomarev, et al., "Development of fast one-dimensional X-ray detector for imaging of explosions," Nucl. Instrum. Meth. Phys., Res. A, 513, Nos. 1-2, 383-393 (2003).

13. K. A. Ten, O. V. Evdokov, I. L. Zhogin, et al., "Measurements of density distributions in detonation processes using synchrotron radiation," Preprint No. 2005-30, Institute of Nuclear Physics, Sib. Div., Russian Acad. of Sci., Novosibirsk (2005).

14. A. N. Dremin, S. D. Savrov, V. S. Trofimov, and K. K. Shvedov, Detonation Waves in Condensed Media [in Russian], Nauka, Moscow (1970).

15. M. A. Cook, The Science of High Explosives, New York (1958).

16. A. V. Fedorov, "Structure and parameters of the detonation-wave front of condensed explosives," Khim. Fiz., 21, No. 8, 66-71 (2002).

17. C. L. Mader, Numerical Modeling of Detonation, Univ. Calif. Press, Berkeley (1979).

18. V. N. Zubarev, N. V. Panov, and G. S Telegin, "Width of the stationary zone in detonation waves," Combust., Expl., Shock Waves, 6, No. 1, 102-106 (1970).

19. L. A. Merzhievskii, V. P. Efremov, P. I. Zubkov, et al., "Synchrotron radiation study of the dynamics of shock-wave compression of an aerogel. Extreme states of Matter. Detonation. Shock waves," in: VII Khariton's Topical Scientific Readings, Proc. Sci. Conf., Sarov (2005), pp. 665-669. 